Ferrata Storti Foundation

\title{
Efficacy of UVC-treated, pathogen-reduced platelets versus untreated platelets: a randomized controlled non-inferiority trial
}

Haematologica 2021

Volume 106(4):1086-1096

\section{Correspondence:}

AXEL SELTSAM

a.seltsam@blutspendedienst.com

Received: May 26, 2020.

Accepted: November 6, 2020.

Pre-published: February 4, 2021.

https://doi.org/10.3324/haematol.2020.260430

(C)2021 Ferrata Storti Foundation

Material published in Haematologica is covered by copyright. All rights are reserved to the Ferrata Storti Foundation. Use of published material is allowed under the following terms and conditions:

https://creativecommons.org/licenses/by-nc/4.0/legalcode. Copies of published material are allowed for personal or internal use. Sharing published material for non-commercial purposes is subject to the following conditions:

https://creativecommons.org/licenses/by-nc/4.0/legalcode, sect. 3. Reproducing and sharing published material for commercial purposes is not allowed without permission in writing from the publisher.

\author{
Veronika Brixner, ${ }^{1 *}$ Gesine Bug, ${ }^{2 *}$ Petra Pohler, ${ }^{3 *}$ Doris Krämer, ${ }^{4}$ \\ Bernd Metzner, ${ }^{4}$ Andreas Voß,${ }^{4}$ Jochen Casper, ${ }^{4}$ Ulrich Ritter, ${ }^{5}$ Stefan Klein, ${ }^{6}$ \\ Nael Alakel, ${ }^{7}$ Rudolf Peceny, ${ }^{8}$ Hans G. Derigs, ${ }^{9}$ Frank Stegelmann, ${ }^{10}$ \\ Martin Wolf, ${ }^{11}$ Hubert Schrezenmeier, ${ }^{12}$ Thomas Thiele, ${ }^{13}$ Erhard Seifried, ${ }^{1}$ \\ Hans-Hermann Kapels, ${ }^{14}$ Andrea Döscher, ${ }^{14}$ Eduard K. Petershofen, ${ }^{14}$ \\ Thomas H. Müller ${ }^{3}$ and Axel Seltsam ${ }^{3,15}$
}

${ }^{1}$ German Red Cross Blood Transfusion Service and Goethe University Clinics, Frankfurt/Main; ${ }^{2}$ Department of Hematology and Oncology, University Hospital Frankfurt, Goethe University, Frankfurt/Main; ${ }^{3}$ German Red Cross Blood Service NSTOB, Springe; ${ }^{4}$ Department of Oncology and Hematology, University Hospital, Oldenburg; ${ }^{5}$ Department of Hematology and Oncology, Municipal Hospital Bremen, Bremen; ${ }^{6}$ Department of Hematology and Oncology, University Hospital, Mannheim; ${ }^{7}$ Medical Clinic I, Department of Hematology and Oncology, University Hospital, Carl Gustav Carus Faculty of Medicine, Dresden; ${ }^{8}$ Department of Hematology and Oncology, Municipal Hospital, Osnabrück; ' ${ }^{9}$ Medical Clinic I, Department of Hematology and Oncology, Carl Gustav Carus Faculty of Medicine, University Hospital, Dresden; ${ }^{10}$ Department of Internal Medicine III, University Hospital, UIm; ${ }^{11}$ Department of Hematology and Oncology, Municipal Hospital, Kassel; ${ }^{12}$ Institute for Transfusion Medicine, University Hospital UIm, UIm; and Institute for Clinical Transfusion Medicine and Immunogenetics UIm, German Red Cross Blood Service Baden-Württemberg - Hessia, UIm; ${ }^{13}$ Institute for Immunology and Transfusion Medicine, Department of Medicine, University of Greifswald, Greifswald; ${ }^{14}$ German Red Cross Blood Service NSTOB, Oldenburg and ${ }^{15}$ Bavarian Red Cross Blood Service, Nuremberg, Germany

*VB, GB and PP contributed equally as co-first authors

\section{ABSTRACT}

2 athogen reduction (PR) technologies for blood components have been established to reduce the residual risk of known and emerging infectious agents. THERAFLEX UV-Platelets, a novel ultraviolet C (UVC) light-based PR technology for platelet concentrates, works without photoactive substances. This randomized, controlled, double-blind, multicenter, non-inferiority trial was designed to compare the efficacy and safety of UVC-treated platelets to that of untreated platelets in thrombocytopenic patients with hematologic-oncologic diseases. The primary objective was to determine non-inferiority of UVC-treated platelets, assessed by the 1-hour corrected count increment (CCI) in up to eight per-protocol platelet transfusion episodes. Analysis of the 171 eligible patients showed that the defined non-inferiority margin of $30 \%$ of UVC-treated platelets was narrowly missed as the mean differences in 1-hour CCI between standard platelets versus UVC-treated platelets for intention-to-treat and per-protocol analyses were $18.2 \% \quad(95 \%$ Confidence Interval [CI]: 6.4-30.1) and 18.7\% (95\% CI: 6.3-31.1), respectively. In comparison to the control, the UVC group had a 19.2\% lower mean 24-hour CCI and was treated with an about $25 \%$ higher number of platelet units, but the average number of days to the next platelet transfusion did not differ significantly between both treatment groups. The frequency of low-grade adverse events was slightly higher in the UVC group and the frequencies of refractoriness to platelet transfusion, platelet alloimmunization, severe bleeding events, and red blood cell transfusions were comparable between groups. Our study suggests that transfusion of pathogen-reduced platelets produced with the UVC technology is safe but non-inferiority was not demonstrated. (clinicaltrials gov. Identifier: DRKS00011156). 


\section{Introduction}

Improvements in donor screening, Good Manufacturing Practice (GMP) and viral marker testing have significantly reduced the incidence of transfusiontransmitted infections. However, the blood supply remains under threat from various disease-causing agents, including known pathogens which are often not assayed in conventional blood screening protocols, i.e., bacteria and human cytomegalovirus, low-titer viruses that escape detection early after infection, and novel emerging transfusion-transmissible pathogens such as Zika virus and hepatitis $\mathrm{E}$ virus. ${ }^{1,2}$ While techniques reducing the biological activities of pathogens in red blood cells are still under development, techniques for platelet and plasma products have been introduced or are under consideration as additional safety measure in an increasing number of countries. ${ }^{3,4}$ Current methods for platelets use chemical reagents (amotosalen or riboflavin) in combination with ultraviolet (UV) light. ${ }^{5}$ However, chemical additives and/or their photoproducts potentially increase the risk of adverse effects, such as immune reactions or toxicity, especially in polytransfused patients.

The THERAFLEX UV-Platelets (Macopharma, Mouvaux, France) method for pathogen reduction (PR) of platelet products is based on treatment with UVC light alone, without any photoactive substances. ${ }^{6}$ UVC is absorbed by nucleic acids, resulting in the formation of pyrimidine dimers, which block the elongation of nucleic acid transcripts. It was shown that UVC treatment significantly reduces the infectivity of platelet concentrates (PC) contaminated by pathogenic viruses, bacteria and parasites. ${ }^{7-12}$ Moreover, this technique was shown in a mouse model to achieve sufficient white cell inactivation to prevent transfusion-associated graft-versus-host disease (TA-GvHD). ${ }^{13}$ Several studies have shown that UVCtreated platelets meet the quality requirements for PC. ${ }^{14-16}$ This randomized controlled non-inferiority trial evaluated the clinical efficacy and safety of pathogen-reduced PC produced using UVC illumination technology compared to that of conventional untreated PCs in thrombocytopenic hematology-oncology patients.

\section{Methods}

\section{Study design}

CAPTURE (Clinical Assessment of Platelets Treated with UVC in Relation to Established Preparations) was designed as a randomized, double-blind, parallel controlled, non-inferiority trial. The trial protocol was approved by a the central ethic committee. Ten hematology/oncology centers in Germany participated in the study. The treatment period started on the day of the first study platelet transfusion and continued for a maximum of 28 days. A safety follow-up period began after the treatment period and continued for 30 days or until the day of early withdrawal, loss to follow-up, or death of the subject, depending on which occurred first (Online Supplementray Figure S1 in the Online Supplementary Appendix).

Adult patients with hematologic or oncologic diseases and with thrombocytopenia or who were expected to become thrombocytopenic after chemotherapy or due to underlying active disease were eligible to participate in the study if they were expected to receive at least one platelet transfusion. All individuals involved in the clinical care, assessment and trial management of the patients were blinded.

\section{Treatment}

Reference and UVC-treated platelet products were either collected by apheresis or prepared from five buffy coats and resuspended in platelet additive solution (PAS). PR of PC was performed within 6 hours after apheresis platelet collection or 6 hours after preparation of the pooled platelet unit using the THERAFLEX UV-Platelets system. ${ }^{6}$ Reference platelets were left untreated or were $\gamma$-irradiated if indicated. UVC-treated products generally were not $\gamma$-irradiated. Patients in both treatment arms received platelet transfusions prophylactically (trigger 10,000/ $\mu \mathrm{L}$ ) or for treatment of bleeding.

\section{Study endpoints}

The primary efficacy endpoint, the 1-hour CCI, was measured 30-90 minutes post-transfusion for each of a maximum of eight per-protocol platelet transfusion episodes per patient administered within the treatment period. The patient's pre-transfusion platelet count, which was used for this calculation, had to be measured within 12 hours before the start of transfusion. CCI was calculated using the formula:

$$
\begin{gathered}
\text { CCI=Post-precount } \times 10^{9} \text { L Platelet dose } \\
\text { tranfused } \times 10^{11} \times \text { BSA } \\
\text { BSA (Body Surface Area })=0.20247 \times \text { Height } \\
\text { m } 0.725 \times \text { Weight } \mathrm{kg} 0.425
\end{gathered}
$$

Secondary efficacy endpoints included the variables 24-hour CCI, 1-hour count increment (CI), 24-hour CI (specimens collected 18-30 hours post-transfusion were considered time compliant), number of platelet transfusions per patient, number of red blood cell (RBC) transfusions per patient, and interval between study platelet transfusions. Secondary safety endpoints included adverse events, clinical and immunological refractoriness, severe bleeding events (World Health Organization [WHO] grade 3 and 4), and alloimmunization to UVC-induced neoantigens on platelets.

\section{Statistical analyses}

The study was designed as a one-sided non-inferiority trial comparing the 1-hour CCI of UVC-treated PC transfusions with those of untreated PC transfusions to test the null-hypothesis and demonstrate that pathogen-reduced platelets are non-inferior to control platelets. The non-inferiority criterion was met if the upper limit of the $95 \%$ Confidence Interval (CI) of the mean difference in 1-hour CCI between the control and UVC groups was below the lower limit of the zone of non-inferiority (based on the results of previous studies with alternative PR methods ${ }^{17-25}$ an up to $30 \%$ reduction of 1 -hour CCI was considered non-inferior). A total of 166 patients were required (83 per arm).

\section{Results}

\section{Patients}

Out of a total of 177 patients screened at 10 study sites between October 2016 and January 2019, 175 were enrolled in the study and randomized to the UVC arm $(n=89)$ or control arm $(n=86)$ (Figure 1). Two patients in each arm did not receive the first platelet transfusion within the specified time period of 6 weeks after randomization and were excluded from the study. The intention-totreat (ITT) analyses were thus performed on 171 patients. After excluding patients who received off-protocol transfusions and/or study platelet transfusions from the wrong treatment arm or who met exclusion criteria, the data set 
Table 1. Baseline characteristics of study patients.

\begin{tabular}{|c|c|c|c|c|}
\hline Parameter & & UVC & Control & $P$ \\
\hline Patients & $\mathrm{n}$ & 87 & 84 & \\
\hline Male/female & $\mathrm{n} / \mathrm{n}$ & $55 / 32$ & $52 / 32$ & 0.876 \\
\hline Age & Years, mean \pm SD & $56.67 \pm 14.11$ & $54.79 \pm 11.90$ & 0.348 \\
\hline Ethnicity & & & & 0.377 \\
\hline Caucasian & n $(\%)$ & $85(97.70)$ & $84(100.00)$ & \\
\hline Asian & n $(\%)$ & $1(1.15)$ & $0(0.00)$ & \\
\hline Other & n (\%) & $1(1.15)$ & $0(0.00)$ & \\
\hline Body surface area & $\mathrm{m}^{2}$, mean $\pm \mathrm{SD}$ & $1.95 \pm 0.20$ & $1.97 \pm 0.23$ & 0.525 \\
\hline Previous pregnancy & n/total n of women (\%) & 27/32 (84.38) & 23/32 (71.88) & 0.365 \\
\hline \multicolumn{5}{|l|}{ Treatment modality } \\
\hline Inpatients & n $(\%)$ & $86(98.85)$ & $83(98.81)$ & 1.000 \\
\hline Outpatients & n $(\%)$ & $1(1.15)$ & $1(1.19)$ & \\
\hline Primary diagnosis & & & & 0.105 \\
\hline Acute lymphoblastic leukemia & n (\%) & $7(8.05)$ & $2(2.38)$ & \\
\hline Acute myeloid leukemia & n $(\%)$ & $40(45.98)$ & $30(35.71)$ & \\
\hline Chronic leukemia & n $(\%)$ & $1(1.15)$ & $0(0.00)$ & \\
\hline Multiple myeloma & n (\%) & $22(25.29)$ & $26(30.95)$ & \\
\hline Non-Hodgkin lymphoma & n (\%) & $9(10.34)$ & $16(19.05)$ & \\
\hline Hodgkin lymphoma & n $(\%)$ & $0(0.00)$ & $3(3.57)$ & \\
\hline Other & n $(\%)$ & $8(9.20)$ & $7(8.33)$ & \\
\hline Treatment & & & & 0.118 \\
\hline Autologous stem cell transplantation & n $(\%)$ & $29(33.33)$ & $41(48.81)$ & \\
\hline Allogenic stem cell transplantation & n $(\%)$ & $6(6.90)$ & $5(5.95)$ & \\
\hline Chemotherapy only & n $(\%)$ & $52(59.77)$ & $38(45.24)$ & \\
\hline \multicolumn{5}{|l|}{ Transfusion history } \\
\hline Platelets & n $(\%)$ & $55(63.22)$ & $45(53.57)$ & 0.217 \\
\hline Red blood cells & $\mathrm{n}(\%)$ & $54(62.07)$ & $57(67.86)$ & 0.522 \\
\hline \multicolumn{5}{|c|}{ Laboratory values prior (<12 hours) to $1^{\text {st }}$ transfusion } \\
\hline Platelet count & $10^{9} / \mathrm{L}$, mean $\pm \mathrm{SD}$ & $59.05 \pm 75.21$ & $52.04 \pm 61.05$ & \\
\hline Hemoglobin & $\mathrm{g} / \mathrm{L}$, mean $\pm \mathrm{SD}$ & $91.15 \pm 14.04$ & $93.54 \pm 13.92$ & \\
\hline International normalized ratio & mean \pm SD & $1.02 \pm 0.11$ & $1.04 \pm 0.15$ & \\
\hline Activated partial thromboplastin time & $\mathrm{s}$, mean $\pm \mathrm{SD}$ & $28.66 \pm 5.98$ & $28.44 \pm 5.28$ & \\
\hline Prothrombin time & $\%$, mean \pm SD & $97.41 \pm 15.49$ & $97.79 \pm 16.44$ & \\
\hline Fibrinogen & $\mathrm{g} / \mathrm{L}$, mean $\pm \mathrm{SD}$ & $3.16 \pm 1.23$ & $3.29 \pm 0.90$ & \\
\hline D-Dimer & $\mathrm{ng} / \mathrm{mL}$, mean $\pm \mathrm{SD}$ & $2935.75 \pm 4086.45$ & $1347.63 \pm 357.09$ & \\
\hline
\end{tabular}

UVC: ultraviolet C; SD: standard deviation: INR: international normailzed ratio; s: seconds.

for the PP analyses consisted of 146 patients. One UVC arm patient who withdrew his informed consent after the first platelet transfusion but agreed to further documentation of adverse events was included in the ITT and PP populations. The planned safety follow-up could not be carried out in a total of six patients. There were no significant differences in the patient characteristics of the two study groups (Table 1).

\section{Transfusions}

In the ITT set, a total of 568 platelet units were transfused, 320 to patients in the UVC arm and 248 to those in the control arm. In the PP set, a total of 432 platelet units were transfused, 249 to patients in the UVC arm and 183 to those in the control arm (Tables 2-4). Most of the transfusions were platelets administered as single units given prophylactically and were performed with apheresis platelet units due to higher recruitment rates in study centers that were using apheresis platelets only (Table 2). The mean pre-transfusion platelet count was about $12 \times 10^{9} / \mathrm{L}$ and did not differ between the two arms. In the control arm, the majority of transfused platelet units (ITT: 77.8\%; PP: $78.1 \%$ ) were $\gamma$-irradiated. Only $4 \%$ of platelet transfusions in the UVC arm and 5\% in the control arm were offprotocol transfusions (Online Supplementary Table S1). Platelet characteristics were similar between study arms.

\section{Platelet transfusion efficacy}

All patients in the UVC arm and 96\% (81 of 84) of the controls were evaluable for analysis in the ITT population. The mean 1-hour CCI value, the primary outcome, was $12.70 \%$ (95\% CI: $11.42-13.97)$ in the UVC group and $15.53 \%$ (95\% CI: 14.18-16.88) in the control group. The mean difference in 1-hour CCI between the control and 
Table 2. Platelet transfusion characteristics and pre-transfusion count (based on intention-to-treat anlysis).

\begin{tabular}{|c|c|c|c|c|}
\hline Parameter & & UVC & Control & $\boldsymbol{P}$ \\
\hline Platelet transfusions & $\mathrm{n}$ & 320 & 248 & 0.041 \\
\hline Apheresis platelets & $\mathrm{n}$ & 223 & 166 & 0.060 \\
\hline Buffy-coat platelets & $\mathrm{n}$ & 97 & 82 & 0.396 \\
\hline Transfusion episodes* & $\mathrm{n}$ & 316 & 245 & 0.038 \\
\hline Single dose & n (\%) & $312(98.73)$ & $242(98.78)$ & \\
\hline Multi-dose & n (\%) & $4(1.27)$ & $3(1.22)$ & \\
\hline Indication for platelet transfusion $^{\dagger}$ & & & & 0.211 \\
\hline Trigger based & n (\%) & $302(98.69)$ & $236(100.00)$ & \\
\hline Prior to intervention & n (\%) & $3(0.98)$ & $0(0.00)$ & \\
\hline Treatment of active bleeding & n (\%) & $1(0.33)$ & $0(0.00)$ & \\
\hline $\mathrm{ABO}$ incompatibility ${ }^{\dagger}$ & & & & 0.353 \\
\hline Major & n (\%) & $32(10.46)$ & $22(9.32)$ & \\
\hline Minor & n (\%) & $82(26.80)$ & $52(22.03)$ & \\
\hline Major and minor & n (\%) & $10(3.27)$ & $13(5.51)$ & \\
\hline No mismatch & $\mathrm{n}(\%)$ & $182(59.48)$ & 149 (63.14) & \\
\hline Platelet dose per single transfusion $^{\dagger}$ & $\mathrm{x} 10^{11}$, mean $\pm \mathrm{SD}$ & $3.26 \pm 0.37$ & $3.30 \pm 0.37$ & 0.242 \\
\hline Storage time ${ }^{\dagger}$ & Days, mean \pm SD & $2.87 \pm 1.18$ & $2.93 \pm 1.23$ & 0.488 \\
\hline Mean pre-transfusion platelet count* & $10^{9} / \mathrm{L}$, mean $\pm \mathrm{SD}$ & $12.58 \pm 6.64$ & $12.14 \pm 7.70$ & 0.544 \\
\hline
\end{tabular}

UVC groups was $18.24 \%$ (95\% Cl: 6.40-30.08). For analysis in the PP population, 75 patients in the UVC arm and 71 patients in the control arm were evaluable. The mean 1-hour CCI value was $13.18 \%$ (95\% CI: $11.80-14.56$ ) in the UVC group and $16.21 \%$ (95\% CI: 14.73-17.70) in the control group. The mean difference in 1-hour CCI between the control and UVC groups was 18.70\% (95\% CI: 6.33-31.07). Thus, the upper bounds of the $95 \%$ CI slightly exceeded the specified margin of $30 \%$ with both ITT and PP approaches, indicating that non-inferiority cannot be claimed (Tables 3 -4, Figure 2). Results for all secondary efficacy endpoints are given in Tables 3-4. For the ITT population, the mean values for platelet count increment parameters were lower in the UVC group than in the control group: $18.5 \%$ for 1 -hour CI, $20.4 \%$ for $24-$ hour CI and $19.2 \%$ for 24 -hour CCI. Patients in the UVC arm received about $25 \%$ more platelet transfusions than patients in the control arm. Accordingly, the mean total dose of platelets transfused per patient was significantly higher in the UVC arm than in the control arm. The mean time interval between platelet transfusions and the mean number of RBC transfusions did not differ significantly between arms. Comparable results were obtained for the PP analysis.

\section{Alloimmunization and refractoriness}

Antibodies against platelet antigens were detected in $10.3 \%$ and $8.3 \%$ of patients of the UVC arm and control arm, respectively, prior to the first study platelet transfusion. Platelet antibodies developed in six patients who tested negative at the beginning of the study: one patient in the UVC arm (human leukocyte antigen [HLA] class I) and five patients in the control arm were affected (three HLA class I, one human platelet antigen [HPA], one HLA class I plus HPA; data not shown). The number of refractory episodes and the number of patients with refractory episodes did not differ significantly between groups (Table 5). Immunological refractoriness due to HLA class I anti- bodies was determined in two patients in the UVC arm and one patient in the control arm; the HLA antibodies were detectable prior to the first study platelet transfusion in all three cases. Platelet antibodies to UVC-related neoantigens were not detected in this study.

\section{Safety}

A total of 1,374 adverse events were documented, 741 in the UVC arm and 633 in the control arm (Table 6). At least one adverse event occurred in 85 patients in the UVC group and in 80 patients in the control arm (Online Supplementary Table S2). The majority of adverse events were non-serious grade 1 and 2 events that were unrelated to the platelet transfusions. The number of mild grade 1 and 2 non-serious adverse events related to platelet transfusion was significantly higher in the UVC arm than in the control arm. The difference between arms was still of only borderline significance when we compared the ratios per platelet transfusion and patient that were calculated to account for the higher number of platelet transfusions in the UVC arm. The symptoms of the reported transfusionrelated adverse events were mainly those known to be associated with platelet transfusions, such as chills, pyrexia, hypersensitivity (allergic reactions), refractoriness and rash (Online Supplementary Table S3). Ten serious adverse events were recorded in each treatment arm; they affected ten patients in the UVC arm and eight in the control arm; none of these serious adverse events were related to the platelet transfusions. Severe bleeding (WHO grade 3 and 4) was observed in one patient in the control arm but in none in the UVC arm. There was no statistically significant difference in mortality between arms (Table 6).

\section{Discussion}

This multicenter, randomized controlled study was designed to evaluate the efficacy and safety of pathogen- 


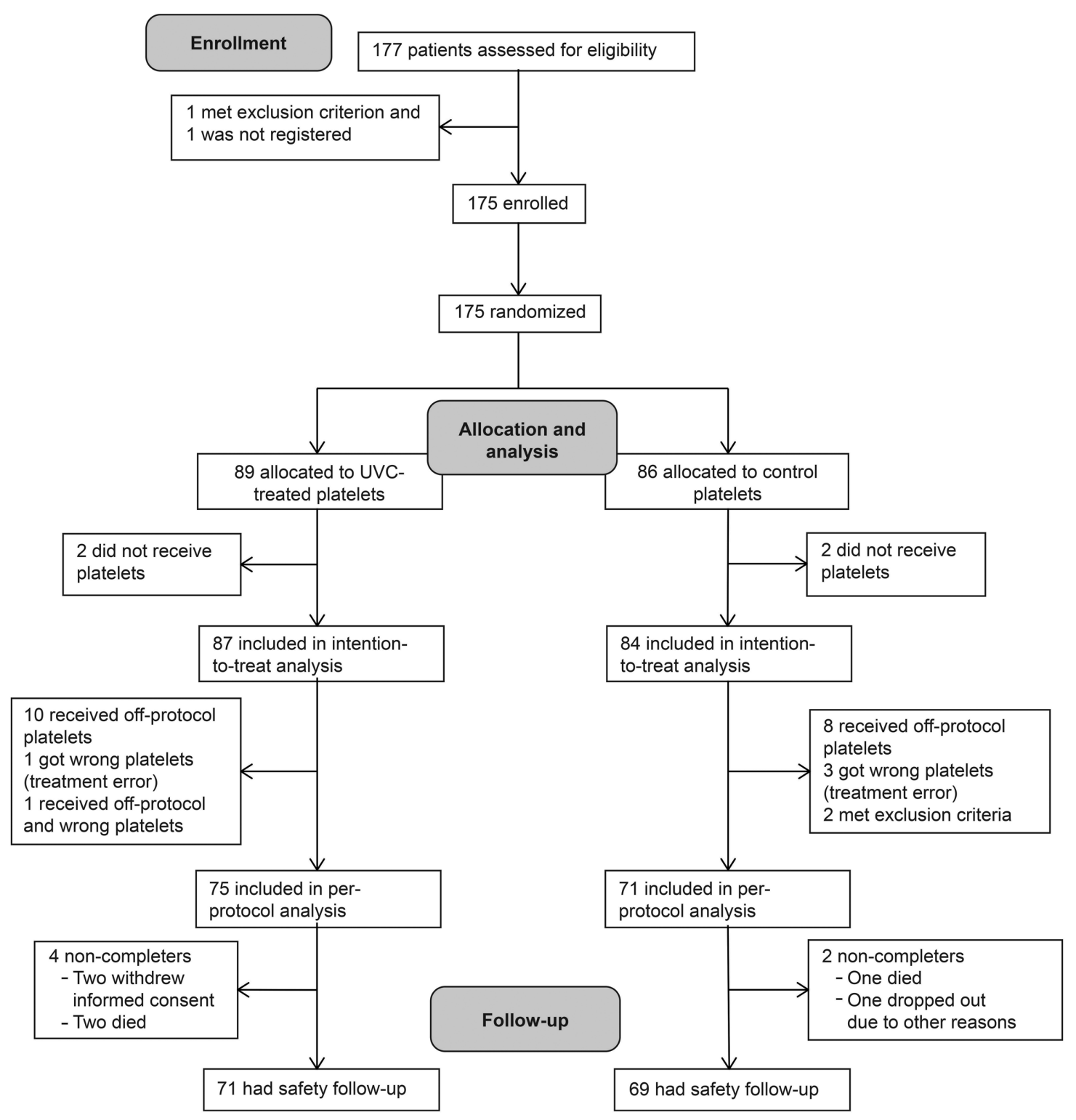

Figure 1. CAPTURE/CONSORT study flow diagram. Off-protocol platelet transfusions were defined as transfusions of conventional platelet units, and treatment errors were defined as transfusions with study platelet products from the wrong treatment arm.

reduced platelets produced by THERAFLEX UV-Platelets PR technology in thrombocytopenic patients with hematologic or oncologic malignancies. One-hour CCI was frequently used as a primary or secondary outcome in previous clinical studies with standard and pathogen-reduced platelets. ${ }^{17-26}$ With one exception, ${ }^{24}$ the transfusion of pathogen-reduced platelets in these studies resulted in a reduction of mean 1-hour CCI, ranging from $12 \%$ to $31 \%$ for amotosalen/UVA-treated PC and from $30 \%$ to $38 \%$ for riboflavin/UV-treated PC. Based on these findings obtained with two alternative PR methods, we set the non-inferiority margin for the mean 1-hour CCI at 30\%. This margin is supported by the result of the PLADO trial investigating single transfusions with platelet doses between $1.1 \times 10^{11}$ and $4.4 \times 10^{11}$ per square meter of bodysurface area in the prophylactic transfusion setting. ${ }^{26} \mathrm{The}$ median 4-hour post transfusion CCI in the low dose platelet group of PLADO was 10 (interquartile range, 5-15) and in the medium dose group also 10 (interquartile range, 6-16). The frequency and severity of bleeding events (WHO grade $\geq 2$ ) in both groups were not higher than that in patients in the high dose group. With a mean platelet dose of $1.7 \times 10^{11} / \mathrm{m}^{2}$ in pathogen-reduced PC in our trial, a CCI reduction by $30 \%$ corresponds to a mean dose of $1.2 \times 10^{11} / \mathrm{m}^{2}$ which is still within the range of the PLADO trial. The about $18 \%$ lower mean 1 -hour CCI, which is very consistent with the result of a radiolabeling study, ${ }^{16}$ and the 19\% lower mean 24-hour CCI in our study suggest a reduced transfusion efficacy for UVC-treated platelets compared to untreated platelets. There are other product-related factors such as the use of a platelet additive solution (PAS) and $\gamma$-irradiation that also affect CCI outcome. ${ }^{24,27}$ In our study, UVC-treated platelets were generally not $\gamma$-irradiated and PAS was used for preparation of both test and control platelet units. Therefore, the lower CCI for UVC-treated platelet transfusions are likely due to 
Table 3. Efficacy endpoints (based on intention-to-treat analysis).

\begin{tabular}{|c|c|c|c|c|}
\hline \multicolumn{2}{|l|}{ Parameter } & \multirow{2}{*}{$\begin{array}{l}\text { UVC } \\
87\end{array}$} & \multirow{2}{*}{$\begin{array}{c}\text { Control } \\
84\end{array}$} & \multirow[t]{2}{*}{$P$} \\
\hline Patients & n & & & \\
\hline Platelet transfusions & $\mathrm{n}$ & 320 & 248 & 0.041 \\
\hline Platelet transfusion episodes* & $\mathrm{n}$ & 316 & 245 & 0.038 \\
\hline \multicolumn{5}{|l|}{ Primary endpoint ${ }^{\dagger}$} \\
\hline \multirow[t]{4}{*}{ 1-hour CCI } & mean \pm SD & $12.70 \pm 5.98$ & $15.53 \pm 6.09$ & \\
\hline & CI $95 \%$ & $11.42-13.97$ & $14.18-16.88$ & \\
\hline & mean difference (CI 95\%) & $2.83(0.99-4.67)$ & & \\
\hline & mean difference (\%) (CI 95\%) & $18.24(6.40-30.08)$ & & \\
\hline \multicolumn{5}{|l|}{ Secondary endpoints ${ }^{\dagger}$} \\
\hline \multirow[t]{2}{*}{ 1-hour CI } & mean $\pm \mathrm{SD}$ & $22.05 \pm 11.35$ & $27.06 \pm 12.25$ & \\
\hline & CI $95 \%$ & $19.63-24.47$ & $24.35-29.76$ & \\
\hline \multirow[t]{2}{*}{ 24-hour CI } & mean \pm SD & $15.07 \pm 9.65$ & $18.94 \pm 11.69$ & \\
\hline & CI 95\% & $13.01-17.12$ & $16.37-21.51$ & \\
\hline \multirow[t]{2}{*}{ 24-hour CCI } & mean $\pm \mathrm{SD}$ & $8.77 \pm 5.52$ & $10.85 \pm 6.16$ & \\
\hline & CI $95 \%$ & $7.59-9.94$ & $9.50-12.21$ & \\
\hline Platelet transfusions per patient & $\mathrm{n}$, mean $\pm \mathrm{SD}$ & $3.68 \pm 2.38$ & $2.95 \pm 2.22$ & 0.041 \\
\hline Platelet transfusion episodes per patient* & $\mathrm{n}$, mean $\pm \mathrm{SD}$ & $3.47 \pm 2.16$ & $2.77 \pm 1.95$ & 0.030 \\
\hline Total dose of platelets transfused per patient ${ }^{\star}$ & $\mathrm{x} 10^{11}$, mean $\pm \mathrm{SD}$ & $11.45 \pm 7.23$ & $9.27 \pm 6.50$ & 0.040 \\
\hline Interval between platelet transfusions & Days, mean \pm SD & $2.62 \pm 1.75$ & $2.80 \pm 1.97$ & 0.586 \\
\hline Red cell transfusions per patient & $\mathrm{n}$, mean $\pm \mathrm{SD}$ & $2.71 \pm 2.39$ & $2.20 \pm 2.37$ & 0.163 \\
\hline
\end{tabular}

${ }^{*}$ Transfusion episode, two or more platelet transfusions on the same day, whereby the interval between two consecutive transfusions is less than 2 hours: ${ }^{\dagger}$ The mean corrected count increment $(\mathrm{CCI})$ and count increment $(\mathrm{CI})$ values were calculated as the mean of the average $\mathrm{CCI} / \mathrm{CI}$ of all transfusions per patient. $\ddagger$ Only per-protocol transfusions were included in this analysis. UVC: ultraviolet C; SD: standard deviation; CI: Confidence Interval.

effects on the platelets, such as increased activation, as described for the other PR methods. ${ }^{28}$

Despite the difference in mean 1-hour CCI of less than $20 \%$ between control and UVC-treated platelets, the noninferiority margin of $30 \%$ was narrowly missed with upper bounds of $30.1 \%$ for the ITT-analysis and $31.1 \%$ for the PP-analysis. Although the 1-hour CCI results of this study do not allow to claim non-inferiority of UVC-treated platelets compared to untreated reference platelets, they are well within the range of those reported for the other PR methods. ${ }^{29}$ In addition, despite lower mean posttransfusion count increments of UVC-treated platelets, the mean 1-hour and 24-hour CCI values are far above the thresholds that have been established to define successful transfusion. $^{30}$

As platelet transfusions are used to treat and prevent bleeding, there is an obvious need for clinical trials of pathogen-reduced platelet products to assess their efficacy with regard to clinically relevant bleeding. However, the results of previous studies consistently suggest that it is probably unlikely that transfusion studies comparing the clinical efficacy of two different platelet preparations can show a significant difference in the prevention of clinically relevant bleeding unless the products differ substantially. ${ }^{5}$ The PLADO trial demonstrated that when following a prophylactic platelet transfusion strategy, which is still the standard of care for most hematology-oncology patients, products with reduced count increments may increase the transfusion frequency but do not necessarily increase the number of clinically relevant bleeding events. ${ }^{26}$ In addition, even large studies investigating prophylactic versus therapeutic platelet transfusion therapy for hematological cancers in up to 300 patients per arm were too small to detect differences in clinically more relevant bleeding of
WHO grade 3 and $4.31,32$ All completed studies of pathogen-reduced platelets, including our trial, followed a prophylactic transfusion regimen and tested products with count increments that were lower than those of the reference product. As expected, a recent Cochrane review and two recently published clinical trials investigating the effectiveness of pathogen-reduced platelets for the prevention of bleeding did not find a difference in the risk of developing clinically severe bleeding compared to standard platelets, although a slight increase in clinically irrelevant WHO grade 2 bleeding was detected in patients in the PR arms. ${ }^{25,33,34}$

It is an ongoing discussion whether CCI can be used as surrogate efficacy marker in platelet transfusion studies. ${ }^{29}$ However, in the absence of a suitable relevant bleeding outcome for platelet transfusion studies, we decided to use the 1-hour CCI as primary efficacy endpoint in our study. ${ }^{35}$ It is at least a measure for the availability of circulating platelets and was used in almost all previous clinical studies with pathogen-reduced platelets, allowing comparison between the different products. ${ }^{29}$

In accordance with previous studies of pathogenreduced platelets, $17,18,20,23,25,34$ the transfusion of UVC-treated platelets was associated with an increased rate of platelet product utilization. The explanation for the higher usage of pathogen-reduced platelet products compared to the reference products may be that, due to a lower platelet increment, the transfusion trigger was met sooner. It remains to be elucidated whether the lower levels of recovery of pathogen-reduced platelets in the circulation could be the result of the early removal of damaged platelets or of the rapid utilization of activated platelets at sites of injury. However, the fact that there was no significant difference in RBC usage between study arms sug- 
Table 4. Efficacy endpoints (based on per-protocol anlysis).

\begin{tabular}{|c|c|c|c|c|}
\hline \multicolumn{2}{|l|}{ Parameter } & \multirow{2}{*}{$\begin{array}{l}\text { UVC } \\
75\end{array}$} & \multirow{2}{*}{$\begin{array}{c}\text { Control } \\
71\end{array}$} & \multirow[t]{2}{*}{$P$} \\
\hline Patients & $\mathrm{n}$ & & & \\
\hline Platelet transfusions & $\mathrm{n}$ & 249 & 183 & 0.030 \\
\hline Platelet transfusion episodes* & $n$ & 245 & 181 & 0.028 \\
\hline \multicolumn{5}{|l|}{ Primary endpoint ${ }^{\dagger}$} \\
\hline \multirow[t]{4}{*}{ 1-hour CCI } & mean $\pm \mathrm{SD}$ & $13.18 \pm 5.98$ & $16.21 \pm 6.18$ & \\
\hline & CI 95\% & $11.80-14.56$ & $14.73-17.70$ & \\
\hline & mean difference (CI 95\%) & $3.03(1.03-5.04)$ & & \\
\hline & mean difference (\%) (CI 95\%) & $18.70(6.33-31.07)$ & & \\
\hline \multicolumn{5}{|l|}{ Secondary endpoints ${ }^{\dagger}$} \\
\hline \multirow[t]{2}{*}{1 hour-CI } & mean $\pm S D$ & $22.94 \pm 11.23$ & $28.36 \pm 12.65$ & \\
\hline & CI 95\% & $20.36-25.53$ & $25.33-31.40$ & \\
\hline \multirow[t]{2}{*}{ 24-hour CI } & mean $\pm \mathrm{SD}$ & $15.76 \pm 9.82$ & $20.37 \pm 11.96$ & \\
\hline & CI $95 \%$ & $13.50-18.02$ & $17.50-23.24$ & \\
\hline \multirow[t]{2}{*}{ 24-hour CCI } & mean $\pm \mathrm{SD}$ & $9.18 \pm 5.65$ & $11.62 \pm 6.24$ & \\
\hline & CI 95\% & $7.88-10.47$ & $10.12-13.12$ & \\
\hline Platelet transfusions per patient & $\mathrm{n}$, mean $\pm \mathrm{SD}$ & $3.32 \pm 2.22$ & $2.58 \pm 1.85$ & 0.030 \\
\hline Platelet transfusion episodes per patient* & $\mathrm{n}$, mean $\pm \mathrm{SD}$ & $3.27 \pm 2.10$ & $2.55 \pm 1.79$ & 0.028 \\
\hline Total dose of platelets transfused per patient ${ }^{\ddagger}$ & $\mathrm{x} 10^{11}$, mean $\pm \mathrm{SD}$ & $10.82 \pm 7.13$ & $8.54 \pm 6.04$ & 0.040 \\
\hline Interval between platelet transfusions & Days, mean \pm SD & $2.70 \pm 1.80$ & $3.06 \pm 2.14$ & 0.372 \\
\hline Red cell transfusions per patient & $\mathrm{n}$, mean $\pm \mathrm{SD}$ & $2.47 \pm 2.19$ & $2.10 \pm 2.25$ & 0.312 \\
\hline
\end{tabular}

Table 5. Refractoriness to platelet transfusions (based on intention-to-treat analysis).

\begin{tabular}{|c|c|c|c|c|}
\hline Parameter & & UVC & Control & $P$ \\
\hline Refractory episodes* & $\mathrm{n}$ & 15 & 6 & 0.055 \\
\hline Patients with at least one refractory episode & $\mathrm{n}(\%)$ & $14(16.09)$ & $6(7.14)$ & 0.095 \\
\hline Single episode of refractoriness & n (\%) & $13(92.86)$ & $6(100.00)$ & \\
\hline Multiple episodes of refractoriness & $\mathrm{n}(\%)$ & $1(7.14)$ & $0(0.00)$ & \\
\hline Immunological refractoriness $^{\dagger}$ & n (\%) & $2(14.29)$ & $1(16.67)$ & 1.000 \\
\hline Antibodies to HLA class I & $\mathrm{n}(\%)$ & $2(100.00)$ & $1(100.00)$ & \\
\hline Antibodies to HPA & n (\%) & $0(0.00)$ & $0(0.00)$ & \\
\hline Antibodies to UVC-related neoantigens & $\mathrm{n}(\%)$ & $0(0.00)$ & $0(0.00)$ & \\
\hline
\end{tabular}

*Episode of "clinical" refractoriness, defined as two consecutive transfusions, each with a 1-hour $\mathrm{CCI}<7.5$. ${ }^{\dagger}$ Episode of "clinical" refractoriness in the presence of platelet antibodies. No immunologic refractoriness due to seroconversion was recorded. UVC: ultrviolet C; HLA: human leukocyte antigen; HLP: human platelet antigen.

gests that the hemostatic function of UVC-treated and reference platelets was equivalent.

An increase in the utilization of platelet units due to reduced increments of pathogen-reduced platelets would have clinically and economically relevant effects. While randomized controlled clinical trials consistently report that pathogen-reduced platelets are associated with a higher number of transfusions per patient, surveillance studies did not show increased usage of PC after universal adaption of a routinely used PR technology. ${ }^{36,37}$ This contradictory result may be explained by the fact that PR implementation in routine practice is often associated with changes in PC specifications and platelet supply logistics that can impact platelet quality. In routine practice, the requirement for generally higher platelet contents in pathogen-reduced PC compared to untreated PC may be a feasible strategy to compensate for the lower recovery of pathogen-reduced platelets, although this could require more blood donations in several settings. ${ }^{38}$
Adverse events overall occurred at similar frequencies and severities in the treatment and control groups. In particular, transfusion-related adverse events were infrequent and mainly low-grade, in line with current hemovigilance data. ${ }^{39,40}$ The higher number of such low-grade transfusion-related adverse events in the UVC arm is due to the fact that episodes of platelet refractoriness, which were more frequently observed in patients receiving UVC-treated platelets, were recorded as transfusion-related low grade 1 and 2 adverse events at some study sites. No unusual adverse events were associated with the transfusion of UVC-treated platelets. Rates of platelet antibodies were low and similar in both arms. Most of the patients with platelet antibodies were pre-immunized prior to the first study platelet transfusion. Similar to the findings reported for other DNA-targeted PR systems, UVC treatment was previously shown to impair direct antigen presentation of antigen-presenting cells in PC, which may possibly reduce alloimmunization in transfusion recipients. ${ }^{13}$ 


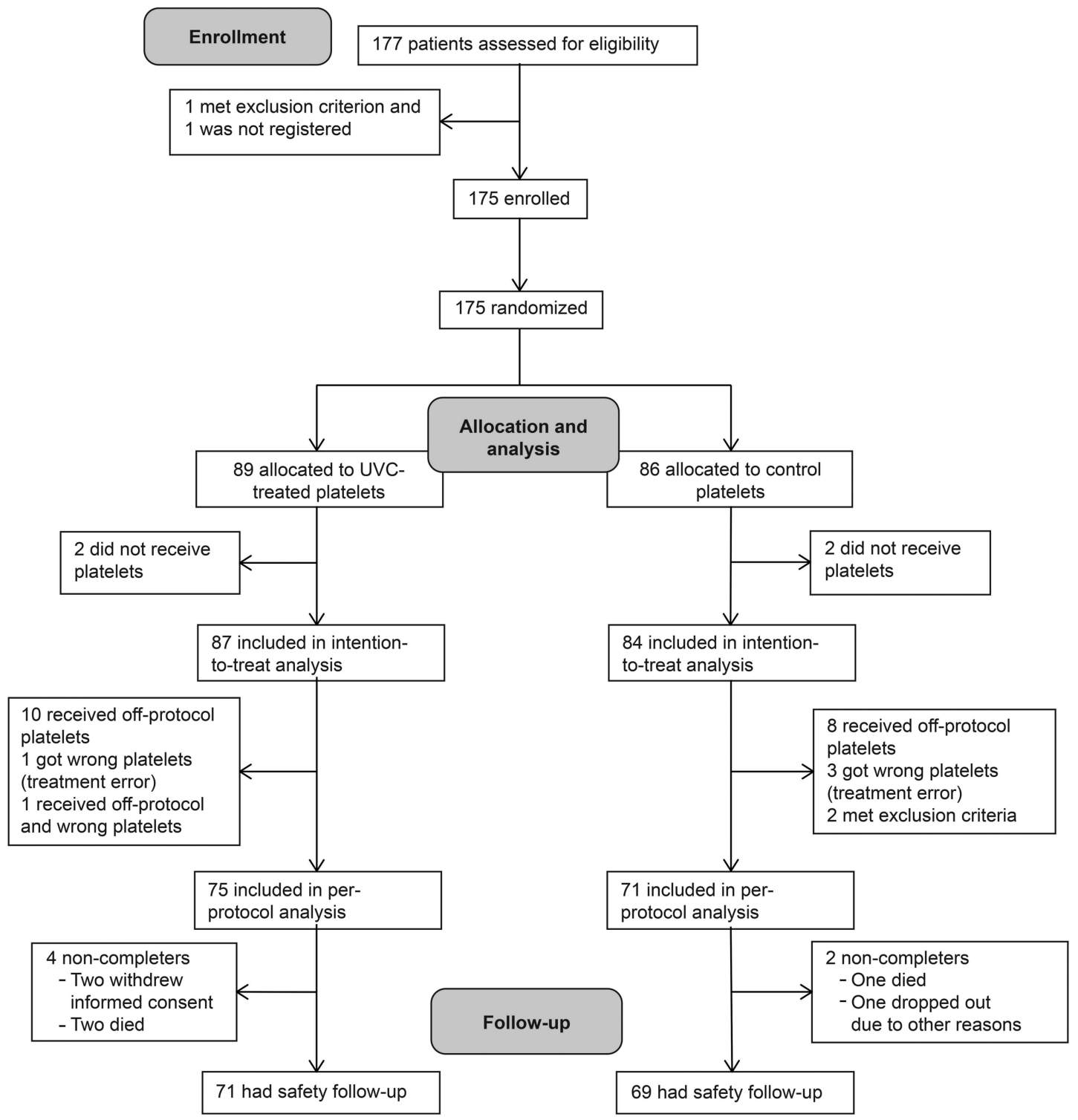

Figure 2. Primary endpoint results. Non-inferiority plot comparing the difference in percentage of the 1-hour corrected count increment (CCI) between the control and UVC (test) arms. The point estimates of the difference in percentage and their 95\% Confidence intervals are displayed for the per-protocol (PP) analysis and the intention-to-treat (ITT) analysis. The dotted vertical line shows the predefined non-inferiority margin of 30.0\%. For both analyses, the $95 \%$ Confidence Interval slightly exceeds the non-inferiority margin.

Reduced immunogenicity of pathogen-reduced treated platelets produced using the riboflavin/UV technology was described in animal studies, but this effect was not observed in clinical studies. ${ }^{41-43}$ The low percentage of immunized patients in our study was too small for any conclusion on the immunogenicity of UVC-treated platelets. A systematic review of the data from 2,075 randomized patients enrolled in 12 studies revealed with high-quality evidence that pathogen-reduced platelets increase the risk of platelet refractoriness in adult cancer patients. ${ }^{33}$ We also found a higher rate of platelet refractoriness in the test arm of our study, which may be explained, at least in part, by the lower mean CCI of the UVC-treated platelets. As also observed for other pathogen-reduced platelets, the lower CCI translates into a higher portion of transfusions with a platelet recovery below the threshold that indicates successful transfusion. ${ }^{30}$

The THERAFLEX UV-Platelets PR technology was developed for platelets suspended in plasma with SSP+PAS, which has been in routine use in Germany for more than a decade. Although limited data is available in the literature, the CCI of platelets stored in this solution seem to be comparable to those of platelets stored in plasma. ${ }^{44}$ Moreover, some evidence obtained with the riboflavin/UV PR system suggests that the use of SSP+ or a similar additive solution protects platelet quality after PR treatment and results in transfusion success rates which are comparable to those of platelets stored in plasma. ${ }^{45,46}$ Thus, we expect that the results obtained with UVC-treated platelets compared to untreated platelets in additive solution may also be extended to the comparison with untreated plasma platelets, which are traditionally used as the reference standard.

There are several limitations of our study. The relationship between CCI and clinically significant bleeding has never been shown. In addition, it is a general limitation to 
Table 6. Adverse events (based on intention-to-treat analysis).

\begin{tabular}{|c|c|c|c|c|}
\hline & & UVC & Control & $\boldsymbol{P}$ \\
\hline Any adverse events* & $\mathrm{n}$ & 741 & 633 & 0.328 \\
\hline Related to platelet transfusion & n (\%) & $34(4.59)$ & $11(1.74)$ & 0.034 \\
\hline Rate per transfusion episode and patient & mean & 0.09 & 0.05 & 0.127 \\
\hline Unrelated to platelet transfusion & n (\%) & $707(95.41)$ & $622(98.26)$ & 0.457 \\
\hline Non-serious adverse events & n (\%) & $731(98.65)$ & $623(98.42)$ & 0.326 \\
\hline Related to platelet transfusion & n (\%) & $34(4.65)$ & $11(1.77)$ & 0.034 \\
\hline Rate per transfusion episode and patient & Mean & 2.69 & 3.38 & 0.159 \\
\hline Unrelated to platelet transfusion & n (\%) & $697(95.35)$ & $612(98.23)$ & 0.454 \\
\hline Grade 1 or 2 adverse events & n (\%) & $617(84.40)$ & $510(81.86)$ & 0.255 \\
\hline Related to platelet transfusion & n (\%) & $33(5.35)$ & $10(1.96)$ & 0.031 \\
\hline Rate per transfusion episode and patient & mean & 0.09 & 0.03 & 0.047 \\
\hline Unrelated to platelet transfusion & n (\%) & $584(94.65)$ & $500(98.04)$ & 0.376 \\
\hline Grade 3 or 4 adverse events & n (\%) & $114(15.60)$ & $113(18.14)$ & 0.895 \\
\hline Related to platelet transfusion & n (\%) & $1(0.88)$ & $1(0.88)$ & 0.987 \\
\hline Unrelated to platelet transfusion & n (\%) & $113(99.12)$ & $112(99.12)$ & 0.897 \\
\hline Serious adverse events & n (\%) & $10(1.35)$ & $10(1.58)$ & \\
\hline Related to platelet transfusion & n (\%) & $0(0.00)$ & $0(0.00)$ & \\
\hline Unrelated to platelet transfusion & n (\%) & $10(100.00)$ & $10(100.00)$ & 0.940 \\
\hline Patients with severe bleeding (WHO grades 3 and 4) & n (\%) & $0(0.00)$ & $1(1.19)$ & 0.491 \\
\hline Death $\mathrm{n}(\%)$ & $2(2.30)$ & $2(2.38)$ & 1.000 & \\
\hline
\end{tabular}

this and other transfusion studies with new platelet products that a consensual non-inferiority margin does not exist. Other trials with pathogen-reduced platelets using the 1-hour CCI as primary endpoint set different non-inferiority margins (e.g., $20 \%$ for the MIRACLE trial). ${ }^{19}$ However, the suitability of bleeding as efficacy outcome in non-inferiority clinical trials with pathogen-reduced platelets is also under debate. ${ }^{35}$ It is a general limitation for clinical trials with pathogen-reduced products that testing the impact of a PR method on blood safety is unfeasible due to the extremely low frequency of infectious transmissions. Nevertheless, the decision to implement a pathogen-reduced product will have to be based on the balance of increased safety for established and emerging pathogens and clinical efficacy, which may be influenced by PR treatment. Randomized controlled trials are generally limited to small numbers of patients and usually focus on patient groups with defined demographic characteristics and treatment indications. In addition, results regarding prophylactic platelet transfusion in adult patients with thrombocytopenia and hematologic diseases are no substitute for clinical studies of pathogen-reduced platelets in pediatric medicine and other clinical contexts, such as post-traumatic coagulopathy. Only postmarketing studies collecting clinical information from standard medical practice in a large number and wide range of patients receiving pathogen-reduced platelet transfusions may allow for a meaningful assessment of rare adverse effects resulting from the use of pathogen-reduced blood products. ${ }^{47}$

\section{Disclosures}

$V B$ received grants from the Research Foundation of the German Red Cross Blood Services (Forschungsgemeinschaft der Blutspendedienste des Deutschen Roten Kreuzes) and
Macopharma for the development of the UVC-based PI technology for platelets and from CERUS for a clinical trial on PI technology for Red Blood Cells. GB received departmental research funding from Novartis, travel grants from Celgene, Gilead, Neovii and Sanofi and honoraria for advisory board memberships for Celgene, Eurocept, Gilead, Hexal, Novartis, Pfizer and Jazz. PP received grants from the Research Foundation of the German Red Cross Blood Services (Forschungsgemeinschaft der Blutspendedienste des Deutschen Roten Kreuzes) and Macopharma for the development of the UVC-based PI technology for platelets. JC received consultancy fee from Pfizer, Merck, Ipsen, Medac; speaker's bureau fees from Pfizer, Merck, Ipsen, Medac; served as consultant for Pfizer, Merck, Ipsen, Medac; received research funding from Ipsen, Medac; reports membership of the Federal Joint Committee (G-BA) Public Policy and Strategy Committee (non-profit). UR received department honoraria for advisory board memberships for Pfizer, Daiichi-Sankyo, Jazz, Servier and Novartis. NA received speaker's bureau fees from Basilea Pharmaceutica, honoraria for advice from Gilead, MSD Sharp \& Dohme GmbH, Pfizer, Amgen and travel grants from Gilead, MSD Sharp \& Dohme GmbH, Pfizer, Amgen. RP received consulting fee from Sanofi Genzyme and Pfizer, and received travel and accommodation expenses from Bristol-Myers Squibb. TT received personal fees from Bristol Myers Squibb, Bayer, Daichii Sankyo, Pfizer, Novo Nordisk, Chugai Pharma and Novartis. THM received grants from the Research Foundation of the German Red Cross Blood Services (Forschungsgemeinschaft der Blutspendedienste des Deutschen Roten Kreuzes) and Macopharma for the development of the UVC-based PI technology for platelets, and reports membership for advisory boards (non-profit) for three German Blood Services (Jena, Rostock, Suhl). AS received grants from the Research Foundation of the German Red Cross Blood Services (Forschungsgemeinschaft der Blutspendedienste des Deutschen 
Roten Kreuzes) and Macopharma for the development of the UVC-based PI technology for platelets, and honoraria for advisory board memberships for Grifols, Quotient and Imusyn. DK, $B M, A V, S K, H G D, F S, M W, H S, E S, H-H K, A D$ and EKP have no conflicts of interest to disclose.

\section{Contributions}

$A S, G B, T H M, P P, V B$ contributed to the conception and design of the study; GB, VB, DK, BM, AV,JC, UR, SK, NA, $R P, H G D, F S, M W, H S, T T, H-H K, A D, E K P$ contributed to the acquisition of data; $A S, T H M, P P$ analyzed and interpreted the data and wrote the manuscript; GB and ES contributed to the interpretation of the data. All authors critically reviewed and revised the manuscript content and checked the final version of the manuscript. All authors are accountable for all aspects of the work.

\section{Acknowledgments}

We are very grateful to all patients who agreed to participate in the CAPTURE trial. Most sincere thanks to Jana Reins, Sabrina Pigur and Doreen Chudziak for study monitoring, to the nursing staff at all participating hospitals for their support, and to Andreas Greinacher (chairman DSMB), Hannes Wandt (member DSMB), and Thomas Kohlmann (member DSMB) for safety oversight. Many thanks to Nancy Heddle for her scientific advice on the design of the study.

Moreover, we wish to thank all blood bank personnel from the German Red Cross Blood Transfusion Service in Frankfurt, Germany and the German Red Cross Blood Service NSTOB in Oldenburg and Springe, Germany for their assistance in the collection, processing, quality monitoring and distribution of the platelet products, and give special thanks to Sarah Dombos, Mesut Karatas, Sebastian Haase, Maren Schepers, Katrin Dahse, Hagen Baume, Ute Gravemann and Anke Wenk. Thanks also to Franz Wagner, Irene Mardt, Nicola Gökbuket and Ina Müller for assessing and processing the serious adverse events.

Thanks to all data managers and statisticians from the Alcedis, Contract Research Organization in Gießen, Germany, particularly Marina Mangold, Sascha Wurmbach, Philipp Schnecko and Claudia Lorenz-Schlüter, and to Macopharma for technical support.

\section{Funding}

This work was supported by the Research Foundation of the German Red Cross Blood Services (Forschungsgemeinschaft der Blutspendedienste des Deutschen Roten Kreuzes) and Macopharma S.A.S. Macopharma had no role in data collection, study management or data analyses.

\section{References}

1. Stramer SL. Current perspectives in transfusion-transmitted infectious diseases: emerging and re-emerging infections. ISBT Sci Ser. 2014;9(1):30-36

2. Lanteri MC, Kleinman SH, Glynn SA, et al. Zika virus: a new threat to the safety of the blood supply with worldwide impact and implications. Transfusion. 2016;56(7):19071914.

3. Jutzi M, Mansouri Taleghani B, Rueesch M, Amsler L, Buser A. Nationwide implementation of pathogen inactivation for all platelet concentrates in Switzerland. Transfus Med Hemother. 2018;45(3):151156.

4. Godbey EA, Thibodeaux SR. Ensuring safety of the blood supply in the United States: donor screening, testing, emerging pathogens, and pathogen inactivation. Semin Hematol. 2019;56(4):229-235.

5. Seltsam A, Muller TH. Update on the use of pathogen-reduced human plasma and platelet concentrates. Br J Haematol. 2013; 162(4):442-454.

6. Seltsam A, Muller TH. UVC irradiation for pathogen reduction of platelet concentrates and plasma. Transfus Med Hemother. 2011;38(1):43-54.

7. Mohr H, Gravemann U, Bayer A, Muller TH. Sterilization of platelet concentrates at production scale by irradiation with shortwave ultraviolet light. Transfusion. 2009;49(9):1956-1963.

8. Castro E, Gonzalez LM, Rubio JM, Ramiro R, Girones N, Montero E. The efficacy of the ultraviolet $C$ pathogen inactivation system in the reduction of Babesia divergens in pooled buffy coat platelets. Transfusion. 2014;54(9):2207-2216.

9. Eickmann M, Gravemann U, Handke W, et al. Inactivation of Ebola virus and Middle East respiratory syndrome coronavirus in platelet concentrates and plasma by ultraviolet $C$ light and methylene blue plus visible light, respectively. Transfusion.
2018:58(9):2202-2207.

10. Faddy HM, Fryk JJ, Prow NA, et al. Inactivation of dengue, chikungunya, and Ross River viruses in platelet concentrates after treatment with ultraviolet C light. Transfusion. 2016;56(6):1548-1555

11. Steinmann E, Gravemann U, Friesland M, et al. Two pathogen reduction technologies--methylene blue plus light and shortwave ultraviolet light--effectively inactivate hepatitis $\mathrm{C}$ virus in blood products. Transfusion. 2013;53(5):1010-1018.

12. Gravemann U, Handke W, Muller TH, Seltsam A. Bacterial inactivation of platelet concentrates with the THERAFLEX UVPlatelets pathogen inactivation system. Transfusion. 2019;59(4):1324-1332.

13. Pohler P, Muller M, Winkler C, et al. Pathogen reduction by ultraviolet $C$ light effectively inactivates human white blood cells in platelet products. Transfusion. 2015;55(2):337-347.

14. Pohler P, Lehmann J, Veneruso V, et al. Evaluation of the tolerability and immunogenicity of ultraviolet C-irradiated autologous platelets in a dog model. Transfusion. 2012;52(11):2414-2426

15. Thiele T, Pohler P, Kohlmann T, et al. Tolerance of platelet concentrates treated with UVC-light only for pathogen reduction--a phase I clinical trial. Vox Sang. 2015;109(1):44-51.

16. Bashir S, Cookson P, Wiltshire M, et al. Pathogen inactivation of platelets using ultraviolet C light: effect on in vitro function and recovery and survival of platelets. Transfusion. 2013;53(5):990-1000.

17. van Rhenen D, Gulliksson H, Cazenave JP, et al. Transfusion of pooled buffy coat platelet components prepared with photochemical pathogen inactivation treatment: the euroSPRITE trial. Blood. 2003;101(6):2426-2433

18. McCullough J, Vesole DH, Benjamin RJ, et al. Therapeutic efficacy and safety of platelets treated with a photochemical process for pathogen inactivation: the SPRINT Trial. Blood. 2004;104(5):1534-
1541

19. Cazenave JP, Folléa G, Bardiaux L, et al. A randomized controlled clinical trial evaluating the performance and safety of platelets treated with MIRASOL pathogen reduction technology. Transfusion. 2010;50(11):23622375.

20. Kerkhoffs JL, van Putten WL, Novotny VM, et al. Clinical effectiveness of leucoreduced, pooled donor platelet concentrates, stored in plasma or additive solution with and without pathogen reduction. $\mathrm{Br}$ J Haematol. 2010;150(11):209-217.

21. Janetzko K, Cazenave JP, Kluter $\mathrm{H}$, et al. Therapeutic efficacy and safety of photochemically treated apheresis platelets processed with an optimized integrated set. Transfusion. 2005;45(9):1443-1452.

22. Lozano M, Knutson F, Tardivel R, et al. A multi-centre study of therapeutic efficacy and safety of platelet components treated with amotosalen and ultraviolet A pathogen inactivation stored for 6 or $7 \mathrm{~d}$ prior to transfusion. $\mathrm{Br} \mathrm{J}$ Haematol. 2011;153(3):393-401.

23. Rebulla P, Vaglio S, Beccaria F, et al. Clinical effectiveness of platelets in additive solution treated with two commercial pathogen-reduction technologies. Transfusion. 2017;57(5):1171-1183.

24. Sigle JP, Infanti L, Studt JD, et al. Comparison of transfusion efficacy of amotosalen-based pathogen-reduced platelet components and gamma-irradiated platelet components. Transfusion. 2013;53(8):17881797.

25. van der Meer PF, Ypma PF, van Geloven N, et al. Hemostatic efficacy of pathogen-inactivated vs untreated platelets: a random ized controlled trial. Blood 2018;132(2):223-231.

26. Slichter SI, Kaufman RM, Assmann SF, et al. Dose of prophylactic platelet transfusions and prevention of hemorrhage. $N$ Engl J Med. 2010;362(7):600-613

27. Seigeot A, Desmarets M, Rumpler A, et al. Factors related to the outcome of prophylactic platelet transfusions in patients with 
